\title{
Relativistic aspects of the JPL planetary ephemeris
}

\author{
W. M. Folkner ${ }^{1}$ \\ ${ }^{1}$ Jet Propulsion Laboratory, California Institue of Technology, \\ 4800 Oak Grove Dr., Pasadena, CA, 91009, USA \\ email: william.m.folkner@jpl.nasa.gov
}

\begin{abstract}
The orbits of the planets as represented by the JPL planetary ephemerides are now primarily determined by radio tracking of spacecraft. Analysis of the data and propagation of the orbits relies on an internally consistent set of equations of motion and propagation of radio signals including relativistic effects at the centimeter level. The planetary ephemeris data set can be used to test some aspects of the underlying theory such as estimates of PPN parameters $\gamma$ and $\beta$, time variation in the gravitational constant $\mathrm{G}$, rotation of the solar system relative to distant objects (Mach's principle), and place stringent limits on the possible violation of the inverse-square law.
\end{abstract}

Keywords. ephemerides, relativity, gravitation

\section{Introduction}

Fitting the orbits of the planets, Sun, and Moon as done in the planetary ephemerides developed at JPL and other institutions must take relativistic effects into account in order to fit the data to the current measurement accuracy. The orbits of the Earth-Moon barycenter and Mars are constrained by copious amounts of radio range measurements to Mars landers and orbiters along with very-long baseline interferometer (VLBI) measurements of Mars orbiters that determine the orbital orientations relative to the IAU celestial reference frame (ICRF). Of the outer planets, Saturn is now best determined through the use of radio range and VLBI measurements of the Cassini spacecraft. Besides determining the planetary orbits, these data can be used to estimate possible corrections to the nominal relativistic models. Preliminary estimates of gravity parameters of interest from the Mars and Saturn data sets are given below. More thorough analyses are in development.

Data for the orbits of Mercury, Venus and the Moon are also useful for testing relativity on the scale of the solar system. However these data are not discussed here, though they are used to constrain the orbits of those objects.

\section{Solar system models}

The basic equations of motion for the solar system are from Einstein et al. (1938) giving the acceleration of the planets, Sun, and Moon as point masses, which are then numerically integrated. In addition the gravitational effects of the solar oblateness, the effects of more than 300 asteroids, and the tidal interactions of the Earth and Moon are taken into account. The initial conditions (orbital elements) of the planets and Moon are estimated as part of the fitting process. The orbit of the Sun is constrained by the conservation of linear and angular momentum. The mass parameters of the planets are derived from the analysis of spacecraft tracking data taken in orbit or during planetary 
encounters, except for the dwarf planet Pluto for which the mass parameter is estimated from astrometric observations of the Pluto system. The mass parameter of the Sun and the solar oblateness parameter $J_{2}$ are estimated from the ephemeris data.

Because the orbit of Mars is significantly affected by asteroids, the treatment of the asteroid mass parameters has a large effect (up to two orders of magnitude) on the uncertainties in the estimated parameters. For the preliminary estimates given below, we estimate the mass parameters for the 67 asteroids with the largest effect on the Mars orbit as identified in Konopliv et al. (2006). The mass parameters for an additional 276 asteroids are modeled in three taxonomic classes and the mean density for each class estimated, based on a nominal radius for each asteroid determined by infrared photometry (e.g. Matson et al. 1986). The Mars spacecraft data set is not sufficient to produce accurate estimates for all 67 individual asteroids, due to large correlations through the effects of the asteroids on the orbit of Mars. This approach results in significantly larger uncertainties in gravity-related parameters than other works, such as Pitjeva (2004), in which only a few asteroid mass parameters are individually estimated. We feel that the estimation of 67 individual asteroid mass parameters gives a more realistic indication of the uncertainties. With a significant number of asteroid mass parameters now being accurately determined through the analysis of astrometric measurements of asteroids that have undergone deflections from asteroid-asteroid interactions (e.g. Baer \& Chesley 2008), improved results may be expected in the near future.

Our modeling of the propagation of radio signals between Earth tracking stations and spacecraft uses the formalism by Moyer (2000). The orbits of spacecraft about Mars are estimated from radio Doppler measurements with an accuracy better than one meter (e.g. Konopliv et al. 2006). After fitting, the radio range measurements to Mars landers and orbiters have a scatter with root-mean-square residuals of about 2 meters in EarthMars distance. The range measurement scatter is dominated by Earth tracking station calibrations done before each tracking pass. Because this calibration error is common to each range measurement, we use only the average range measurement for each tracking pass. Since there are an average of 70 range measurements per tracking pass, an estimation which includes each range measurement, ignoring the correlated effect due to the calibration process, would result in an estimate too optimistic by almost an order of magnitude.

\section{PPN Parameter estimation}

The parameterized post-Newtonian (PPN) parameters $\gamma$ and $\beta$ characterize a range of possible theories for gravity, with values of unity under Einstein's theory of general relativity.

The PPN parameter $\gamma$ mainly appears in measurements of radio range delay during solar conjunction (e.g. Shapiro 1964). An estimate of $\gamma$ from the effective delay on radio range measurements to the Viking landers during the solar conjunction of 1976 was given with an uncertainty of 0.002 by Reasenberg et al. (1979). From the radio range measurements from the Viking landers during the solar conjunctions of 1976 and 1979, combined with radio range and VLBI measurements from later orbiting spacecraft not during conjunction, we find that $(\gamma-1)=0.00009 \pm 0.00070$. This uncertainty is larger than that given by Pitjeva (2004) mainly due to our treatment of the uncertainties in the asteroid mass parameters. In normal ephemeris development we have not previously included radio range measurements from recent Mars orbiting spacecraft near solar conjunction due to concerns about possible correlations of the solar plasma delay with other parameters such as PPN $\gamma$. In a preliminary look at radio range measurements during the 
Mars conjunction of 2009 we find that the solar plasma and PPN $\gamma$ effects are reasonably de-correlated and get an improved estimate of $(\gamma-1)=0.00030 \pm 0.00050$. A better estimate has been given from an experiment with Cassini using a multi-band radio experiment to cancel the solar plasma with an uncertainty a factor of 25 lower by Bertotti et al. (2003).

The PPN parameter $\beta$ mainly appears in the Mars spacecraft data set through the precession of the perihelion of Mars, an effect predicted by general relativity to be $\delta \phi /$ orbit $=(2-\beta-2 \gamma) 6 \pi \mathrm{M}_{\odot} /\left[3 \mathrm{a}\left(1-\mathrm{e}^{2}\right)\right]$ with a nominal value of $1.35 \%$ / century. With the current Mars spacecraft data set we find $(2 \gamma-\beta-1)=+0.0004 \pm 0.0012$. The uncertainty is larger than some other published values due to our treatment of uncertainties in the asteroid mass parameters. An analysis of radio range measurement to the Venus Express orbiter may be expected to give a better constraint due to less perturbations by the asteroids. A tighter constraint on $\beta$ has been derived from lunar laser ranging analysis (e.g. Williams et al. 2004).

\section{Inverse-square law}

Analysis of radio tracking data of some spacecraft appear to give a small anomalous acceleration of the spacecraft radially toward from the Sun (e.g. Anderson et al. 1998). It has been recognized that radio range measurements to Mars from the Viking landers show no such anomalous acceleration at a much lower level. From the current Mars radio range data set, we find upper bounds on a radial acceleration of Earth and Mars to be less than $3 \times 10^{-14} \mathrm{~m} / \mathrm{s}^{2}$ and $8 \times 10^{-14} \mathrm{~m} / \mathrm{s}^{2}$ respectively. A tighter constraint on such an effect can be derived from radio tracking data of the Cassini spacecraft in orbit about Saturn, giving a radial acceleration of Saturn as $<1 \times 10^{-14} \mathrm{~m} / \mathrm{s}^{2}$.

Another common test is to look for a time variation in the gravitation constant $G$. From the Mars spacecraft data set we can estimate a time variation in the mass parameter of the Sun. We find that $1 /\left(G M_{\odot}\right) \times d\left(G M_{\odot}\right) / d t<2 \times 10^{-13} /$ year, comparable to the uncertainty given from lunar laser ranging by Williams et al. (2004). By comparison, the mass loss of the Sun due to emission of photons is about $7 \times 10^{-14} /$ year. Assuming the mass loss from the Sun is comparable with that from photon emission, the estimate of the rate of change of the Sun's mass parameter gives an upper bound on the rate of change in the gravitation constant $G$. We have looked at the Mars spacecraft data set to see if it can be used to separately distinguish a rate of change of the Sun's mass and the gravitation constant $G$ and we find that the two are almost completely correlated and hence not distinguishable.

\section{Mach's principle}

The orbits of the planets are integrated in a locally inertial (non-rotating) reference frame through use of the Einstein-Infeld-Hofmann equations of motion. Lunar laser ranging and radio ranging to Mars-orbiting spacecraft determine the internal dynamics of the solar system to very high accuracy. For example, the mean motion of Mars relative to Earth can be determined with an accuracy of about 0.001 " / century without use of any observations relative to objects outside the solar system. The dynamical ephemeris is usually aligned to an external coordinate system through use of angular measurements of planets or spacecraft in orbit about planets relative to stars or extra-galactic radio sources. We normally assume that the locally inertial reference frame is not rotating with respect to the rest of the universe. This assumption (Mach's principle) can be tested by 
comparing the mean motion of Mars relative to Earth determined from ranging measurements with the mean motion determined from VLBI observations of Mars-orbiting spacecraft. In a preliminary assessment of this effect we find that the dynamical rotation rate of the solar system relative to extra-galactic radio sources is less than 0.004 " / century. This upper bound is larger than the expected rotation rate of the solar system about the galactic center. The estimate could be affected by systematic errors in the catalog of extra-galactic radio sources as well as by limits on modeling the motions of Earth and Mars. The uncertainty in the rate is expected to improve in the near future with updates in the catalogs of radio sources and subsequent reprocessing of Mars spacecraft VLBI measurements in the coming year.

\section{Acknowledgements}

The results presented here are based on the enormous efforts of reducing spacecraft orbits relative to the planets and other aspects of radio measurement processing, that were provided by Alex Konopliv, Robert Jacobson, and James Border of JPL and Trevor Morely and colleagues at ESOC. This research was carried out in part at the Jet Propulsion Laboratory, California Institute of Technology, under contract with the National Aeronautics and Space Administration.

\section{References}

Anderson, J. D., Laing, P. A., Lau, E. L., Liu, A. S., Nieto, M. M., \& Turyshev, S. G. 1998, Phys. Rev. Lett., 81, 2858

Baer, J. \& Chesley, S. R. 2008, Celest. Mech. Dyn. Astr., 100, 27

Bertotti, B., Iess, L., \& Tortora, P. 2003, Nature, 425, 374

Einstein, A., Infeld, L., \& Hoffmann, B. 1938, Ann. Math, 39, 65

Konopliv, A. S., Yoder, C. F., Standish, E. M.,Yuan, D. N., \& Sjogren, W. L. 2006, Icarus, 182, 23

Matson, D. L., Veeder, G. J., Tedesco, E. F., Lebronsky, L. A. \& Walker, R. G. 1986, Adv. Space Res. 6(7), 47

Moyer, T. D. 2000, Formulation for Observed and Computed Values of Deep Space Network Data Types for Navigation JPL Publication 00-7 (Pasadena: Jet Propulsion Laboratory, California Institute of Technology)

Pitjeva, E. V. 2004, Astron. Lett., 31, 340

Reasenberg, R. D., Shapiro, I. I., MacNeil, P. E., Goldstein, R. B., Briedenthal, J. C., Brinkle, J. P., Cain, D. I., Kaufman, T. M., Komarek, T. A., \& Zygielbaum, A. I. 1979, ApJ, 234, $\mathrm{L} 219$

Shapiro, I. I. 1964, Phys. Rev. Lett., 13, 789

Williams, J. G., Turyshev, S. G., \& Boggs, D. H. 2004, Phys. Rev. Lett., 93, 261101 